\title{
The Baikal neutrino experiment
}

\author{
A. Avrorin a, V. Aynutdinov ${ }^{\mathrm{a}, *}$, V. Balkanov ${ }^{\mathrm{a}}, \mathrm{I}_{\text {. Belolaptikov }}{ }^{\mathrm{d}}, \mathrm{S}$. Berezhnev ${ }^{\mathrm{c}}$, D. Bogorodsky $^{\mathrm{b}}$, \\ N. Budnev ${ }^{\text {b }, ~ I . ~ D a n i l c h e n k o ~}{ }^{a}$, G. Domogatsky ${ }^{a}$, A. Doroshenko ${ }^{a}$, A. Dyachok $^{\text {b }}$, Zh. Dzhilkibaev $^{\text {a }}$,

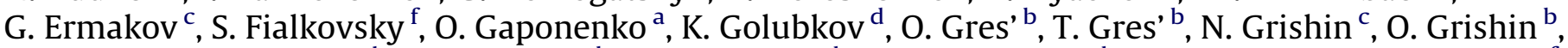 \\ A. Klabukov a , A. Klimov ${ }^{h}$, A. Kochanov ${ }^{b}$, K. Konischev ${ }^{d}$, A. Korobchenko ${ }^{b}$, A. Koshechkin ${ }^{\text {a }}$, V. Kulepov ${ }^{f}$, \\ D. Kuleshov ${ }^{\text {a }}$, L. Kuzmichev ${ }^{\text {c }}$, E. Middell ${ }^{\text {e }}$, S. Mikheyev ${ }^{\mathrm{a}}$, M. Milenin ${ }^{\mathrm{f}}$, R. Mirgazov ${ }^{\mathrm{b}}$, E. Osipova ${ }^{\mathrm{c}}$, \\ A. Pan'kov ${ }^{b}$, L. Pan'kov $^{b}$, A. Panfilov ${ }^{a}$, D. Petukhov ${ }^{a}$, E. Pliskovsky ${ }^{\text {, }}$ P. Pokhil ${ }^{a}$, V. Poleschuk ${ }^{a}$, \\ E. Popova ${ }^{\text {c }, ~ V . ~ P r o s i n ~}{ }^{\text {c }}$, M. Rozanov ${ }^{g}$, V. Rubtzov ${ }^{\text {b }}$, A. Sheifler ${ }^{\text {a }}$, A. Shirokov ${ }^{c}$, B. Shoibonov ${ }^{\text {, }}$, \\ Ch. Spiering ${ }^{\text {e }}$, O. Suvorova ${ }^{\mathrm{a}}$, B. Tarashansky ${ }^{\mathrm{b}}$, R. Wischnewski ${ }^{\mathrm{e}}$, A. Zagorodnikov ${ }^{\mathrm{b}}$, \\ V. Zhukov ${ }^{\mathrm{a}}$, A. Yagunov ${ }^{\mathrm{b}}$
}

anstitute for Nuclear Research, 60th October Anniversary pr. 7a, Moscow 117312, Russia

b Irkutsk State University, Irkutsk, Russia

c Skobeltsyn Institute of Nuclear Physics MSU, Moscow, Russia

d Joint Institute for Nuclear Research, Dubna, Russia

e DESY, Zeuthen, Germany

${ }^{\mathrm{f}}$ Nizhni Novgorod State Technical University, Nizhni Novgorod, Russia

${ }^{\mathrm{g}}$ St. Petersburg State Marine University, St. Petersburg, Russia

${ }^{\mathrm{h}}$ Kurchatov Institute, Moscow, Russia

\section{A R T I C L E I N F O}

Available online 30 June 2010

Keywords:

Neutrino telescopes

Baikal

\begin{abstract}
A B S T R A C T
We review the status of the Lake Baikal Neutrino Experiment. Preparation towards a $\mathrm{km}^{3}$-scale Gigaton Volume Detector (GVD) in Lake Baikal is currently a central activity. As an important milestone, a $\mathrm{km}^{3}$ prototype string comprising of 12 optical modules and based on a completely new technology, has been installed and was put in operation together with NT200+ in April, 2009. We also present recent results from the long-term operation of NT200, including an improved limit on the diffuse astrophysical neutrino flux.
\end{abstract}

(c) 2010 Elsevier B.V. All rights reserved.

\section{Introduction}

The Baikal Neutrino Telescope is operated in Lake Baikal, Siberia, at a depth of $1.1 \mathrm{~km}$. Lake Baikal is one of the most extraordinary lakes in the world. Baikal water is characterized by an absorption length of about $20-25 \mathrm{~m}$ and a scattering length of $30-50 \mathrm{~m}$. Good water properties and the possibility to use ice as a natural platform for detector deployment make the Baikal site very attractive for the creation of a neutrino telescope.

The first stage telescope configuration NT36 was mounted in 1993. It was the first underwater array, and the first neutrino events were detected with this installation. The full physics program was started in 1998 with the telescope configuration NT200 [1,2]. NT200 consists of 8 strings, each with 24 pairwise

\footnotetext{
* Corresponding author. Tel.: +8 49512335 08; fax: +7 4997839298

E-mail address: aynutdin@yandex.ru (V. Aynutdinov).
}

arranged optical modules (OM). Each OM contains a 37-cm diameter hybrid photodetector QUASAR-370, developed specially for this project [3]. The upgraded Baikal telescope NT200+ [4] was commissioned in April, 2005, and is made of a central part (the old, densely instrumented NT200 telescope) and three additional external strings.

The Baikal collaboration follows for several years an R\&D program for a gigaton volume detector (GVD) in Lake Baikal. The construction of NT200+ was a first step towards such a $\mathrm{km}^{3}$-scale neutrino telescope. The most recent $\mathrm{km} 3$-milestones were the construction and installation of new technology prototype strings in 2008 and 2009. The basic goals of the prototype strings installation are investigation and in-situ test of basic elements of the future detector. Fig. 1 gives a sketch of the telescope NT200+, including the new GVD prototype string.

In this talk we will present selected physics results obtained with NT200, describe preliminary design and expected sensitivity of the GVD telescope, and discuss the experience of the GVD prototype string operation. 


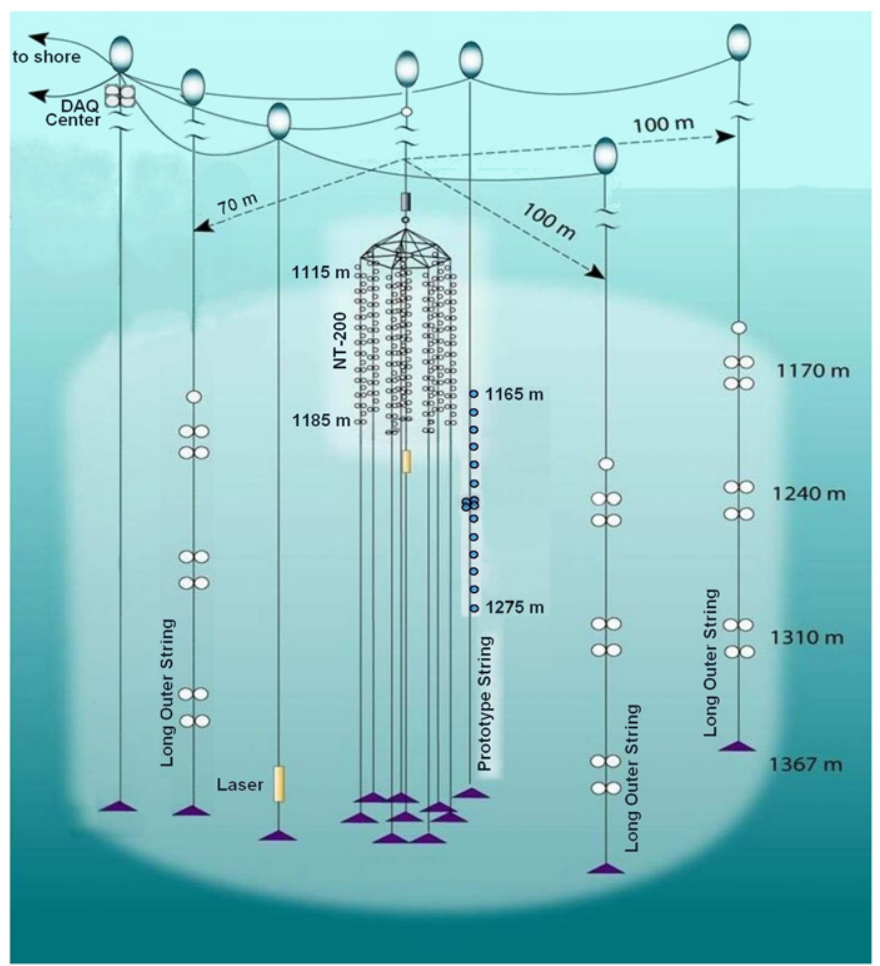

Fig. 1. The Baikal Telescope NT200+ and the GVD prototype string with $12 \mathrm{OM}$.

\section{Recent physics results from NT200}

The physics program of the Baikal experiment covers the typical spectrum of high energy neutrino telescopes [5-10]. In this paper we review selected astroparticle physics results from the long-term operation of NT200, in particular, an improved limit on the diffuse astrophysical neutrino flux, upper limits on the muon flux from annihilation of hypothetical weakly interacting massive particles (WIMPs) in the Sun, and a limit on the neutrino flux associated with gamma-ray bursts.

\subsection{A search for extraterrestrial high-energy neutrinos}

Our search for high energy extraterrestrial neutrinos is based on studies of bright cascades detected in the telescope NT200. A full cascade reconstruction algorithm (for vertex, direction, and cascade energy) was applied to the 1038 live days of data taken with NT200 in 1998-2002. Cuts were then placed on this reconstructed cascade energy to select neutrino-induced events. Within systematic and statistical uncertainties there are no significant excess above the expected background from atmospheric muons (see Fig. 2). For an $E^{-2}$ behaviour of neutrino spectrum a $90 \%$ C.L. upper limit on the neutrino flux of all flavours obtained with the Baikal neutrino telescope NT200 is: $E_{\mathrm{v}}^{2} \Phi<2.9 \times 10^{-7} \mathrm{~cm}^{-2} \mathrm{~s}^{-1} \mathrm{sr}^{-1} \mathrm{GeV}$, for $20 \mathrm{TeV}<E_{\mathrm{v}}<20 \mathrm{PeV}$.

\subsection{A search for WIMP neutrinos from the Sun}

A possible signal from WIMP annihilation in the Sun would appear as an excess of upward going muons over atmospheric neutrinos arriving from the direction of Sun. We have applied two sorts of quality cuts, optimized for high and low WIMP masses. We have selected 510 and 2376 upward going muon candidates in the two data samples for 1007 live days. The distributions of

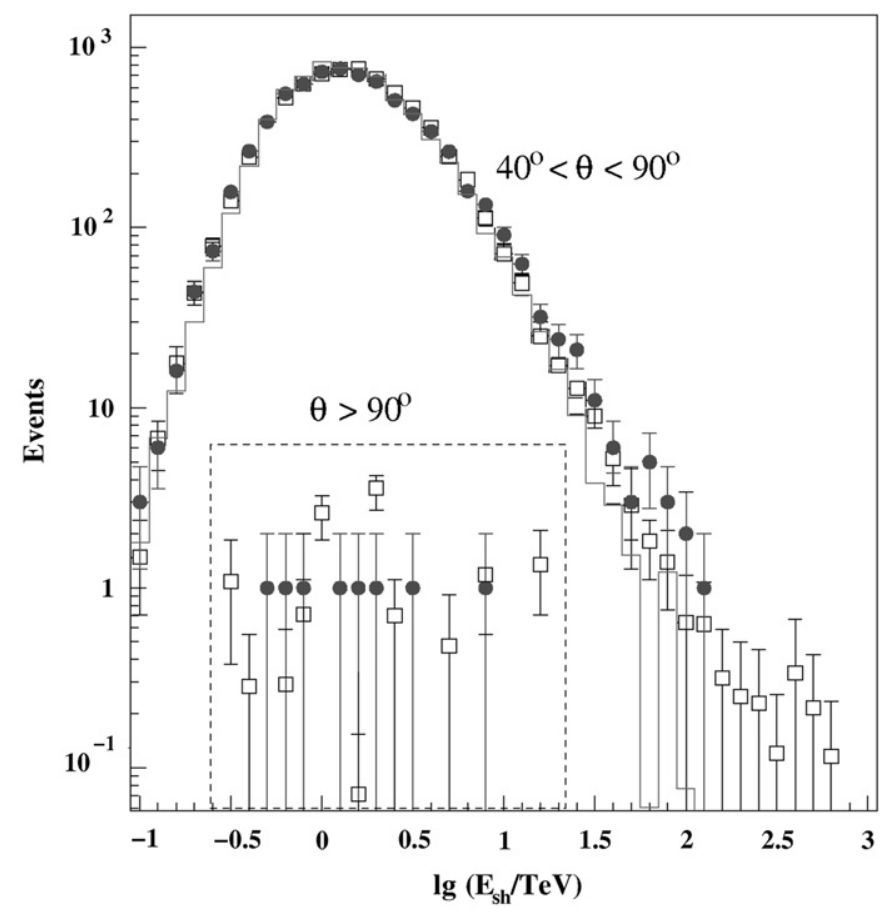

Fig. 2. Reconstructed cascade energy distribution for data (dots) and for MC-generated atmospheric muons (boxes); true MC energy distribution given as histogram.

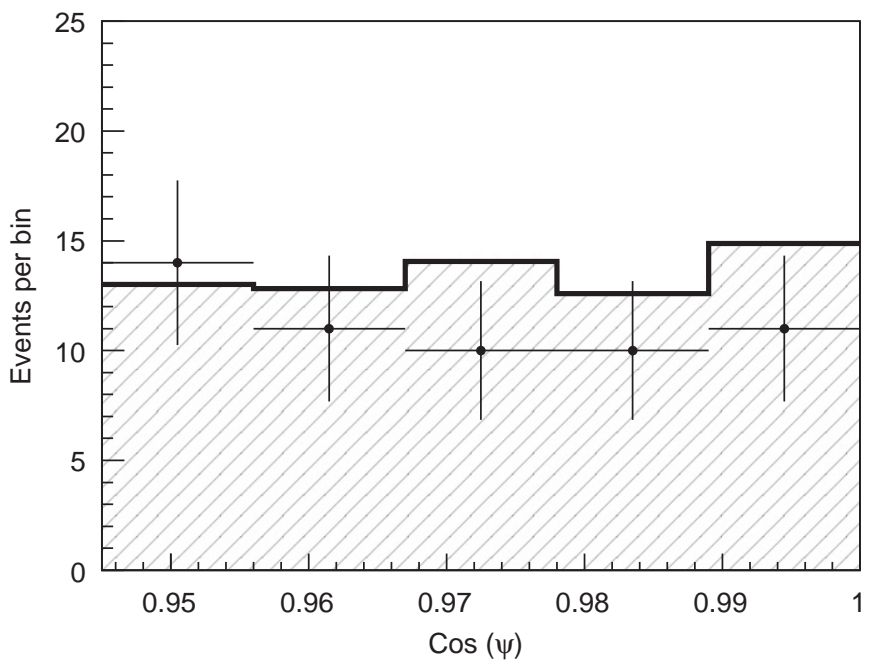

Fig. 3. Mismatch angle $\Psi$ (Muon/Sun): data and background (histogram).

correlation angles between these muons and the Sun were compared to the corresponding off-source background expectation. In Fig. 3 we show the results for the sample of larger statistics.

No indications for excess muons were found. The 90\% C.L. upper limits on the muon flux from the Sun are obtained as functions of the WIMP mass for $b$ anti- $b$ (soft channel) and $W^{+} W^{-}$ (hard channel) neutrino energy spectrum [11]. For WIMP masses $>500 \mathrm{GeV}$ the limit depends weakly on the WIMP mass and is $\Phi<3 \times 10^{3} \mathrm{~km}^{-2} \mathrm{yr}^{-1}$. The presented results are preliminary, and allow estimating the NT200 sensitivity for high energy neutrinos from DM annihilation processes in the Sun. 


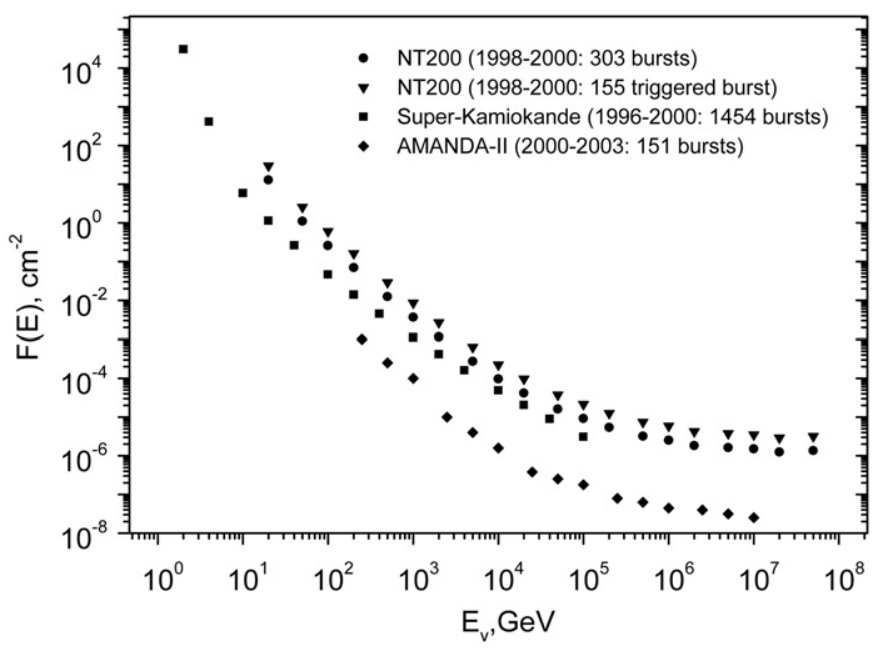

Fig. 4. About $90 \%$ C.L. upper limits on the GRB neutrino fluence Green's function for NT200, Super-Kamiokande [13] and AMANDA [14].

\subsection{A search for neutrinos from gamma-ray bursts}

The detection strategy for neutrino events from gamma ray bursts (GRB) is based on a search for up-going muons correlated with a GRB in time and direction. Information about GRB time and location on the sky allows reducing the atmospheric muon background and, as a result, significantly increases the sensitivity of the neutrino telescope to neutrino events [12]. We have presented the results of a search for neutrino-induced muons detected with NT200 in coincidence with 303 gamma-ray bursts recorded from 1998 to 2000. The sensitive angular range of NT200 covers most part of the Southern hemisphere. No evidence for neutrino-induced muons from gamma-ray bursts is found. The resulting Green's Function fluence limit for this search is presented in Fig. 4.

Our results extend to that of Super-Kamiokande by two-orders of magnitude in energy. Assuming a Waxman-Bahcall neutrino spectrum and using only triggered gamma-ray burst (155 GRB), a neutrino flux upper limit of $E_{v}^{2} \Phi_{v} \leq 1.1 \times 10^{-6} \mathrm{GeV} \mathrm{cm}^{-2} \mathrm{~s}^{-1} \mathrm{sr}^{-1}$ was obtained.

\section{Gigaton volume detector in lake Baikal}

Preparation towards a $\mathrm{km}^{3}$-scale Gigaton Volume Detector (GVD) in Lake Baikal is currently a central activity. In this section we will discuss the results of optimization of the GVD design, basic principles of the organization of the data acquisition system, and results of in-situ tests of the GVD prototype string.

\subsection{Preliminary results of GVD optimization}

The basic approach to design the Baikal $\mathrm{km}^{3}$-detector GVD is the same as to NT200 design. GVD will consist of strings of optical modules that will be grouped in clusters. This approach provides a relatively flexible structure, which allows for a rearrangement of the main building blocks (clusters), to adapt to requirements of new scientific goals, if necessary.

The objective of the GVD design optimization is to provide a maximum cascade detection volume under conditions of effective registration of high energy muons. MC-optimization for the $\mathrm{km} 3$-detector design is going on. The present calculation was performed for 96 strings grouped in 12 clusters with 192 OMs each. Optical modules contain photomultipliers R-7081HQE with

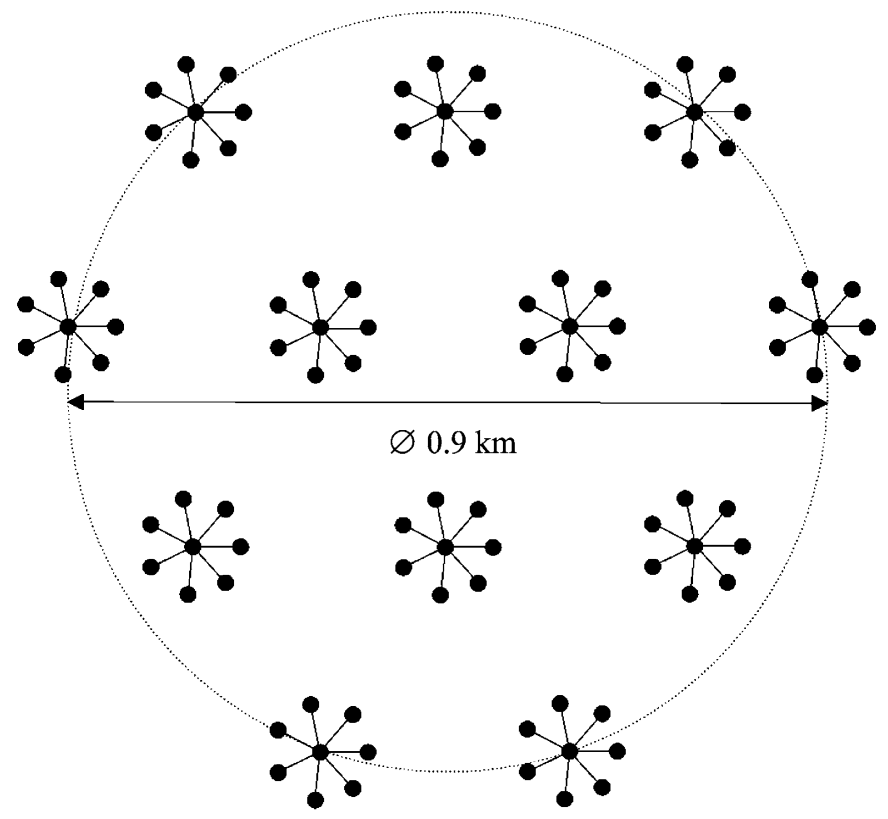

Fig. 5. Top view of GVD: 96 strings are grouped in 12 clusters.

photocathode diameter of $10 \mathrm{in}$. and quantum efficiency of $35 \%$. Basic parameters for the optimization were $Z$-the vertical distance between OMs, $R$-the distance between string and cluster centre, and $H$-the distance between cluster centres. Coincidences of any neighbouring $\mathrm{OM}$ on a string (thresholds 0.5 and 3 p.e.) were used as a trigger condition for this calculation.

The compromise between cascade detection volume and muon effective area was obtained for the following values of the parameters: $H=300 \mathrm{~m}, R=60 \mathrm{~m}$, and $Z=15 \mathrm{~m}$. The preliminary design of GVD is presented in Figs. 5 and 6. It contains a total of 2304 OMs, arranged on 96 strings with 24 OMs each, and an instrumented length of $345 \mathrm{~m}$.

The effective area of installation for muons above $10 \mathrm{TeV}$ with reconstruction error $0.5-l^{\circ}$ is $0.1-1 \mathrm{~km}^{2}$, and the effective volume for cascade events above $50 \mathrm{TeV}$ with reconstruction error $2-4^{\circ}$ is $0.3-0.8 \mathrm{~km}^{3}$.

\subsection{Data acquisition system of GVD}

The data acquisition system of GVD is constructed from three basic building blocks: optical modules (OMs), sections of OMs (main detection units), and clusters.

The OM consists of a photomultiplier (PMT), a high-voltage unit (HV), a two-channel preamplifier, and a controller. The block diagram of the OM electronics is presented in Fig. 7. The photomultiplier R-7081HQE was preliminary selected as light sensor for the OMs. This PMT has a hemispherical photocathode with a diameter of $10 \mathrm{in}$. and a quantum efficiency of about $35 \%$. The OM controller is intended for HV regulation and monitoring, for permanent PM noise measurements, and for time and amplitude calibration of the measuring channel with two internal LED. Slow control data to and from the OMs are transferred via an underwater RS-485 bus.

The OM electronics and the PMT are placed in a pressureresistant $42 \mathrm{~cm}$ diameter glass sphere. Two connectors are placed on the glass sphere for communication. A high permittivity alloy cage surrounds the PMT, shielding it against the Earth's magnetic field. 


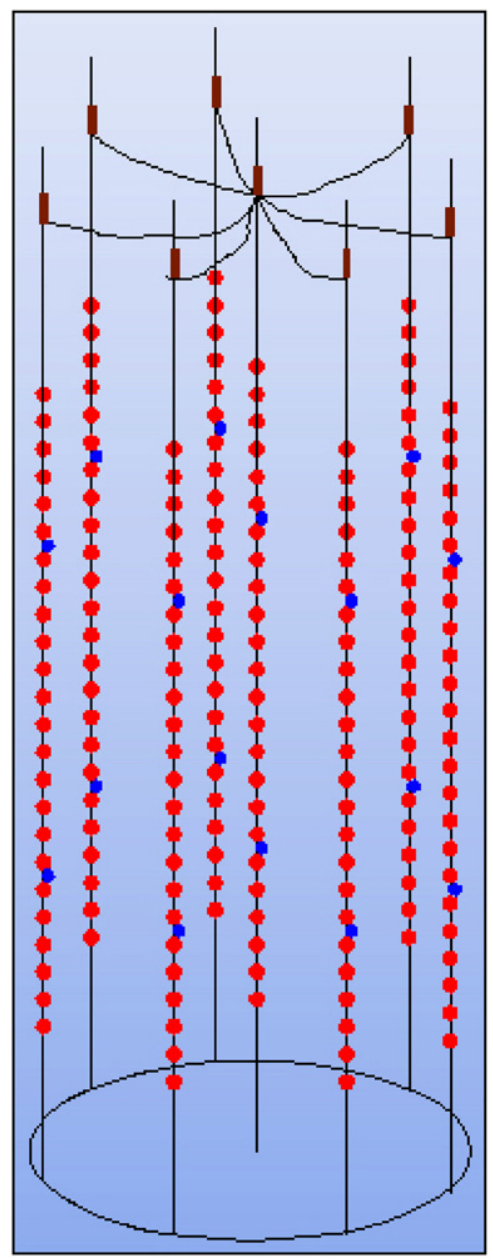

Fig. 6. Schematic view of a cluster, made up of 8 strings with 192 OMs in total.

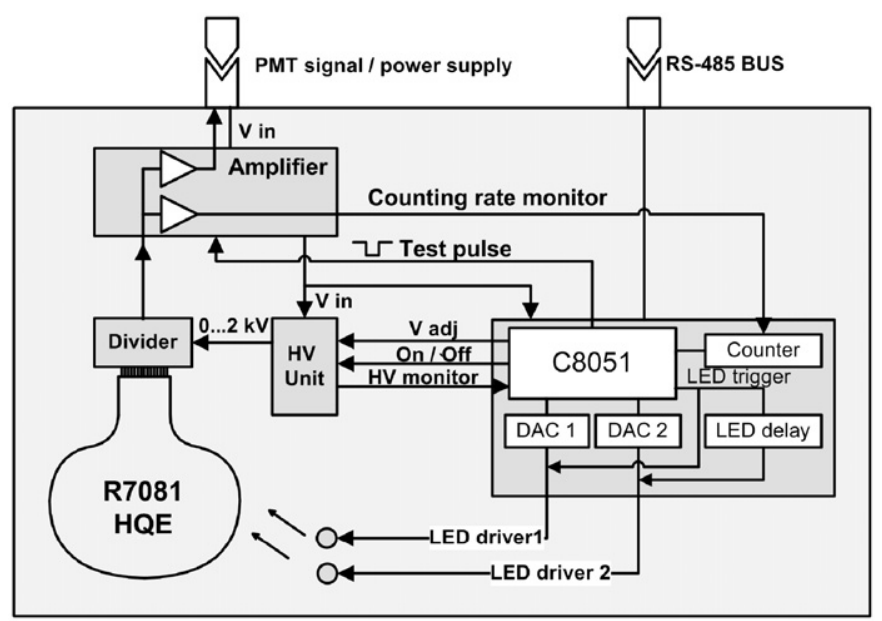

Fig. 7. Functional scheme of the optical module electronics.

Optical modules of each string are grouped into two sections. The functional scheme of one section is presented in Fig. 8. The section is the basic detection unit of GVD. It consists of 12 OMs, the service module (SM), and the electronics unit (BEG) with analog-digital converters on the basis of FADCs. Analog signals from the OM are transmitted to the BEG through coaxial cables of $90 \mathrm{~m}$ length. BEG consists of three FADC boards (12 channels in total), an OM power controller, and a VME controller. The VME

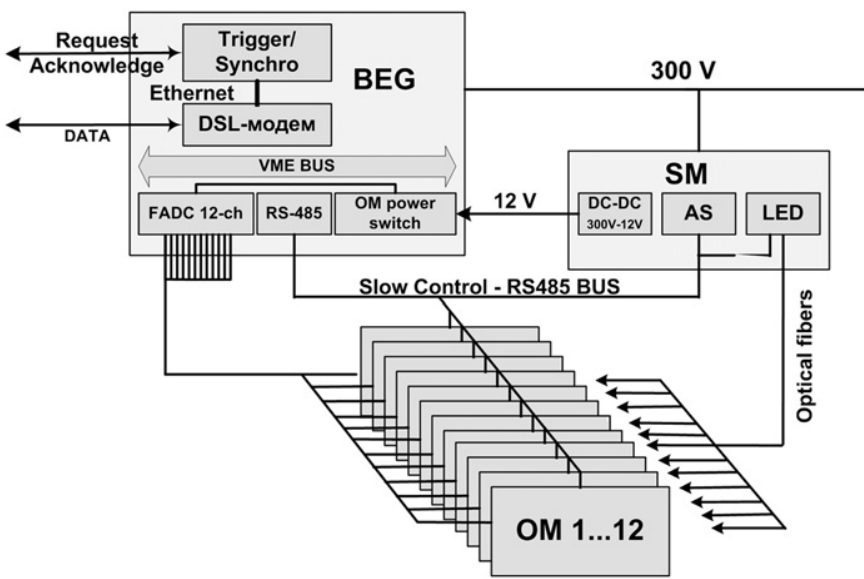

Fig. 8. Functional scheme of the section of the optical modules: basic detection unit of GVD.

controller provides trigger logic, data readout from FADC boards and connection via local Ethernet to the underwater PC on the cluster. The trigger is formed by a coincidence of any neighbouring OMs within a section (thresholds 0.5 and 3 p.e.). Every FADC board consists of four simultaneously digitizing FADCs. Every FADC has 12 bit resolution and samples at $200 \mathrm{MHz}$.

The power control board is intended for the OM power supply. This module allows switching power on/off for each optical module.

Data from the BEG are transmitted via an Ethernet connection (10 Mbit DSL-modem) to the underwater micro-PC of the cluster. Slow control of OMs is provided by BEG via a RS-485 underwater bus.

Time and amplitude calibration of the section are provided by a LED flasher unit located in the service module (SM). Basic elements of the LED flasher unit are two LED drivers and a LED controller. The LED controller has the same design as the OM controller, and provides the possibility of LED pulse amplitude and delay regulation independently for the two LEDs. Light pulses from the flasher are transmitted to each OM via individual optical fibers.

Two sections form the string (24 OM in total). 8 strings are combined to a cluster (see Fig. 6). The cluster DAQ centre provides inter-string time synchronization, on-line data selection, and communication to shore through an optical cable.

\subsection{Prototype string of GVD}

The first prototype of GVD electronics was installed in Lake Baikal in April 2008 [15]. It was a reduced size section with 6 OMs. This unit provided the possibility to study the basic elements of the future detector: new optical modules, the DAQ system of section and the calibrating system.

In April 2009 a prototype of the GVD string with two sections was installed as a part of NT200+ detector. The objectives were studies of the basic DAQ/triggering approach for the $\mathrm{km}^{3}$-detector and investigation of time accuracy of synchronization of the sections. The design of the new prototype string is presented in Fig. 9.

The prototype string consists of 12 optical modules with six photomultipliers R8055 (Hamamatsu, 13 in. photocathode) and six XP1807 (Photonis, 12 in. photocathode), two BEGs, a service module (SM) and a micro-PC unit. The distance between OMs along the string is $10 \mathrm{~m}$. The upper 10 OMs have photomultipliers with downward looking photocathode, while the two bottom OMs contain PMs with upward looking photocathode. Data from the FADC units (BEG) are transmitted via a local Ethernet line to 


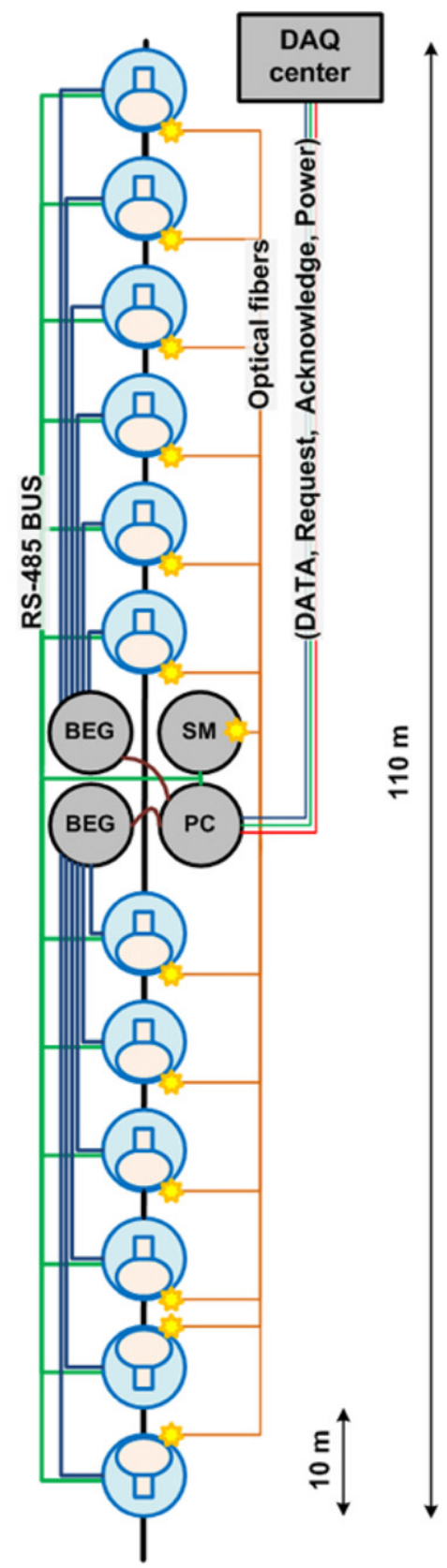

Fig. 9. The km3-prototype string with 12 OMs.

the underwater micro-PC unit that emulates the operation of the future cluster DAQ centre. SM contains the string calibration system on the basis of LEDs (see Section 3.2) and DC-DC converters for OM power supply.

Analysis of data obtained during two months of the string operation is in progress now. Fig. 10 shows an example of the PMT count rates for three channels of the string during this period (threshold of $\sim 0.5 \mathrm{pe}$ ). One can see the strong correlation between count rates of different channels. It indicates that significant part of OM noise is due by the light background of Baikal water.

The basic objective of the present analysis is the investigation of muon detection with the prototype string. For this purpose, time differences $\Delta T$ between muon pulses detected with different channels are studied and compared with simulation of the string response to the atmospheric muon flux. The example of the $\Delta T$-distribution for two up-ward looking PMTs of the string is presented in Fig. 11.

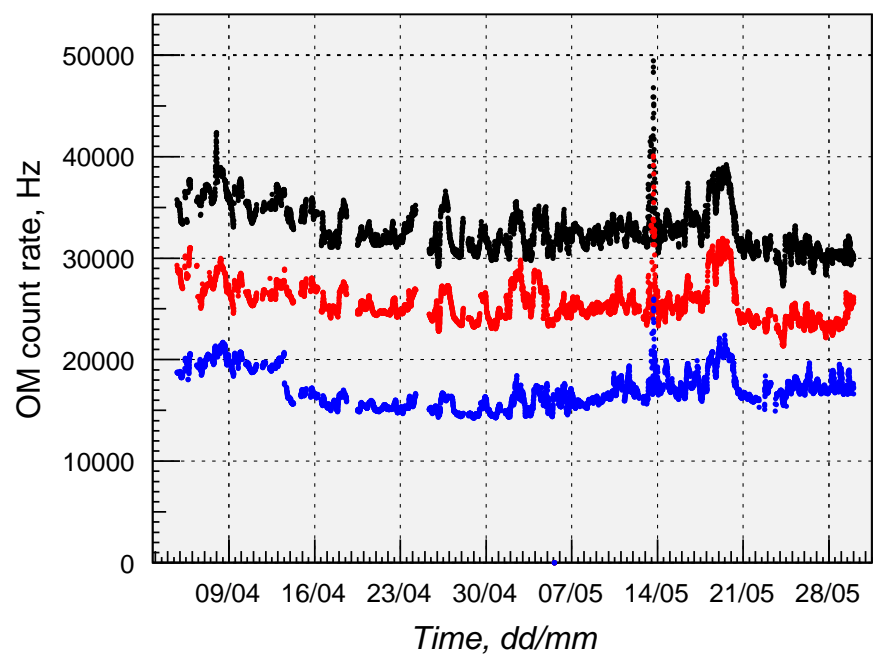

Fig. 10. The PMT count rate for 1 st-3rd channel of the string during two months of exposure.

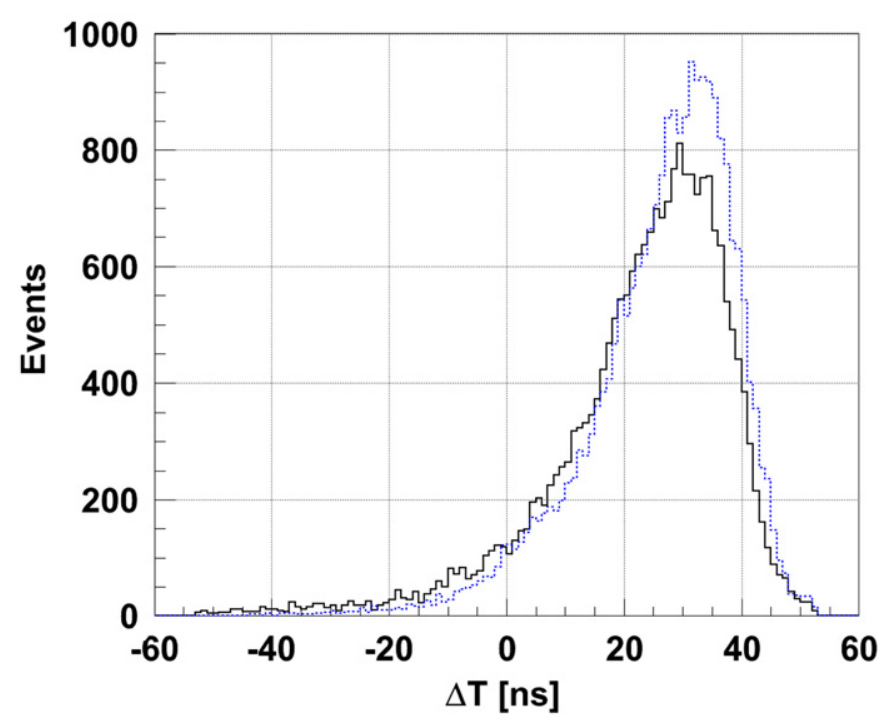

Fig. 11. Distribution of time difference $\Delta T$ between muon pulses for two up-ward looking PMTs: experiment and calculation.

Experimental data are in good agreement with the calculation. But, for down-looking photomultipliers the agreement between experiment and model is somewhat worse. Precise measurements of the angular sensitivity of new OMs are conducted now to clarify this contradiction.

\section{Conclusion}

The Baikal neutrino telescope NT200 is working successfully for more than 10 years. This installation allowed to obtain an upper limit for the diffuse astrophysical neutrino flux on the level $E^{2} \Phi=2.9 \times 10^{-7} \mathrm{~cm}^{-2} \mathrm{~s}^{-1} \mathrm{sr}^{-1} \mathrm{GeV}$. Also, limits on the muon fluxes induced by WIMP annihilation in the Sun have been set. The limit on the neutrino flux from gamma-ray bursts greatly complements existing data for the Southern hemisphere GRB.

Preparation towards a km3-scale Gigaton Volume Detector in Lake Baikal is currently a central activity. For detailed investigations and in-situ tests of new basic elements of the future detector a "new technology" prototype string with six large area hemispherical PMs and with FADC readout technology was designed 
and installed in spring 2008 in Lake Baikal. An upgraded version of the prototype string, which comprises 12 optical modules, is operating in Lake Baikal since April 2009. The km3-detector Technical Design Report is planned for fall 2010.

\section{Acknowledgments}

This work was supported in part by the Russian Ministry of Education and Science (GK 02.740.11.0018, GK 02.518.11.7158, P1242, P2504, RNP 2.2.1.1/1483, RNP 2.1.1/1539, RNP 2.2.1.1/ 5901, REC «BAIKAL»), by the German Ministry of Education and Research, by the Russian Found for Basic Research (Grants 07-0200791, 08-02-00432, 08-02-00198, 08-02-10010, 09-02-10012, 09-02-10001, 09-02-00623, 09-02-12295), by the Grant of the President of Russia NSh-321.2008-2, and by NATO Grant NIG-9811707 (2005).

\section{References}

[1] I. Belolaptikov, et al., Astropart. Phys. 7 (1997) 263.

[2] V. Aynutdinov, et al., Nucl. Instr. and Meth. A588 (2008) 99.

[3] R. Bagduev, et al., Nucl. Instr. and Meth. A420 (1999) 138.

[4] V. Aynutdinov, et al., Nucl. Instr. and Meth. A567 (2006) 433.

[5] A. Avrorin, et al., in: Proceedings of the 31st ICRC, Lodz, Poland, 2009 arXiv:0909.5562.

[6] V. Aynutdinov, et al., Nucl. Instr. and Meth. A602 (2009) 14

[7] V. Aynutdinov, et al., Astropart. Phys. 25 (2006) 140.

[8] A. Avrorin, et al., Astron. Lett. 35 (651) (2009).

[9] V. Aynutdinov, et al., Nucl. Instr. and Meth. A567 (2006) 433.

[10] K. Antipin, et al., in: Proceedings of the 30th ICRC Conference, Merida, Mexico, 2007, arXiv:0710.3063.

[11] A. Avrorin, et al., in: Proceedings of the 31st ICRC Conference, Lodz, Poland 2009, arXiv:0909.5589.

[12] A. Avrorin, et al., in: Proceedings of the 31st ICRC Conference, Lodz, Poland, 2009, arXiv:0910.4327.

[13] S. Fukuda, et al., Astrophys. J. 578 (2002) 317 arXiv:0205.304.

[14] A. Achterberg, et al., Astrophys. J. 674 (2008) 357 arXiv:0705.1186.

[15] V. Aynutdinov, et al., Nucl. Instr. and Meth. A602 (2009) 227 